\title{
Clinical Practice Guideline for Accurate Diagnosis and Effective Treatment of Gastrointestinal Stromal Tumor in Korea
}

\author{
Yoon-Koo Kang, MD, PhD \\ Hye Jin Kang, MD, PhD² \\ Kyoung-Mee Kim, MD, $\mathrm{PhD}^{3}$ \\ Taesung Sohn, MD, PhD ${ }^{4}$ \\ Dongil Choi, MD, PhD \\ Min-Hee Ryu, MD, PhD \\ Woo Ho Kim, MD, PhD ${ }^{6}$ \\ Han-Kwang Yang, MD, $\mathrm{PhD}^{7}$ \\ On Behalf of the Korean GIST Study \\ Group (KGSG)
}

${ }^{1}$ Department of Oncology, Asan Medical Center, University of Ulsan College of Medicine, ${ }^{2}$ Division of Hematology/ Oncology, Department of Internal Medicine, Korea Cancer Center Hospital,

Korea Institute Radiological and Medical Sciences, Departments of ${ }^{3}$ Pathology,

${ }^{4}$ Surgery, and ${ }^{5}$ Radiology, Samsung Medical Center, Sungkyunkwan University School of Medicine, ${ }^{6}$ Department of Pathology,

${ }^{7}$ Department of Surgery \& Cancer Research Institute, Seoul National University College of Medicine, Seoul, Korea
Despite their rarity in incidence and prevalence, gastrointestinal stromal tumors (GISTs) have emerged as a distinct and noteworthy pathogenetic entity. The clinical management of GISTs has rapidly evolved due to the recent elucidation of their oncogenic signal transduction pathway and the introduction of molecular-targeted therapies. Successful management of GISTs requires a multidisciplinary approach firmly based on an accurate histopathologic diagnosis. In 2007, the Korean GIST study group published the first guideline for optimal diagnosis and treatment of GISTs in Korea. The second version of the guideline was published in 2010. Herein, we provide the results of relevant clinical studies for the purpose of further revision to the guideline. We expect this new guideline will enhance the accuracy of diagnosis, as performed by members of the Korean associate of physicians involved in GIST patient care, thus improving the efficacy of treatment.

\section{Key words}

Guideline, Gastrointestinal stromal tumors, Diagnosis, Surgery, Drug therapy, Molecular targeted therapy, Imatinib, Sunitinib

Correspondence: Yoon-Koo Kang, MD, PhD Department of Oncology, Asan Medical Center, University of Ulsan College of Medicine, 88 Olympic-ro 43-gil, Songpa-gu,

Seoul 138-736, Korea

Tel: $82-2-3010-3210$

Fax: 82-2-3010-8772

E-mail: ykkang@amc.seoul.kr

Received March 5, 2012

Accepted April 25, 2012

\section{Introduction}

Gastrointestinal stromal tumors (GISTs) are relatively rare neoplasms occurring in the gastrointestinal (GI) tract, omentum or mesentery. However, they are the most common sarcoma of the GI tract and account for $5 \%$ of all sarcoma cases. Typically, complete surgical resection of the tumor is curative, but recurrence of tumors in the liver or peritoneum are common. Unresectable or recurrent tumors do not respond to conventional cytotoxic chemotherapy and are thus associated with poor patient prognosis. However, the identification of a signal transduction pathway associated with the development of GISTs and the use of molecular-targeted therapy with imatinib (Glivec, Novartis Korea, Seoul, Korea) have yielded remarkable outcomes. Imatinib has been shown to not only prolong 
survival time but also improve quality of life with generally manageable side-effects as compared to conventional cytotoxic chemotherapies. Imatinib is used as a first-line therapy for unresectable and/or metastatic GISTs. Neoadjuvant and adjuvant treatments with imatinib are also being investigated.

The molecular pathologic mechanism of GIST has been elucidated and, in Western countries, histopathologic criteria have recently been standardized. Guidelines incorporating these criteria have been published by the National Comprehensive Cancer Network (NCCN) [1] and the European Society for Medical Oncology (ESMO) [2]. In Japan and Australia, the appropriate guidelines for clinical practice in each country have also been published [3,4]. The Korean GIST Study Group (KGSG), a multidisciplinary group consisting of pathologists, surgeons, gastroenterologists, diagnostic radiologists and medical oncologists which was first assembled in December 2006, published the first guideline for the diagnosis and treatment of GISTs that is suitable for Korean clinical practice in 2007, with a second version in 2010 [5,6]. In this third version of the guideline, we sought to generate updates to topics and add recommendations. In terms of pathologic diagnosis, we added a description of succinate dehydrogenase (SDH)-deficient GISTs. And based on the results of the Scandinavian Sarcoma Group and Sarcoma Group of the Arbeitsgemeinschaft Internistische Onkologie (SSGXVIII/AIO) trial, we recommended that post-operative adjuvant imatinib treatment for at least 36 months should be considered for patients with high-risk GISTs [II, C]. In addition, based on the results from a large-scale retrospective analysis (the KGSG study), we suggested that a high-dose imatinib should be considered for treatment of Korean patients with KIT exon 9 mutations, as recommended for Western patients with a similar genotype [IV, C]. However, this suggestion needs to be rigorously tested.

Expert panel members of the KGSG thoroughly reviewed the relevant literature, including the ESMO and NCCN guidelines, and shared their experiences and opinions in order to form a consensus on twenty topics related to the pathologic diagnosis, and surgical and medical treatment of GISTs. We had not defined the level of evidence for each recommendation in the first and second versions of the guideline, but have added these to this third version of the guideline. Levels of evidence [I-V] and grades of recommendation [A-D], as used by the American Society of Clinical Oncology, are provided in square brackets as seen in Table 1 [7].

The consensus served as the foundation for the guideline of the diagnosis and treatment of patients with GISTs, which hopefully can be used in order to optimize the clinical management of GIST cases in Korea.

\section{Pathologic Diagnosis of GISTs}

\section{Definition of GISTs}

GISTs are the most common mesenchymal tumor of the GI tract [8]. GISTs arise from the interstitial cells of Cajal or their common stem cell [9]. GISTs can arise in any portion of the GI tract, but usually occur in the stomach $(60 \%)$ or small intestine $(30 \%)[10,11]$. GISTs range in size from tiny tumors measuring less than $1 \mathrm{~cm}$, typically discovered incidentally during tests for other diseases, to very large lesions measuring upwards of $35 \mathrm{~cm}$ (median, $5 \mathrm{~cm}$ ) [12]. Irrespective of their size, GISTs share morphologic features and immunoreactivity for KIT, and contain an oncogenic mutation in the KIT (80-85\%) or platelet-derived growth factor receptor (PDGFRA, 5-7\%) genes [13]. Although most GISTs are driven by KIT or PDGFRA-activating mutations, a small subset are associated with loss of function of the SDH complex of the mitochondrial inner membrane proteins. SDH-deficient GISTs in particular include pediatric cases and those associated with Carney triad or Carney-Stratakis syndromes [14].

\section{Pathologic findings of GISTs}

Upon gross examination, a GIST is typically a well-circumscribed fleshy, pink or tan-white mass. Large tumors frequently show hemorrhage,

Table 1. Levels of evidence and grades of recommendation

\begin{tabular}{|c|c|c|c|}
\hline Level & Source of evidence & Grade & Grade of recommendation \\
\hline $\mathrm{I}$ & $\begin{array}{l}\text { Meta-analysis of multiple well-designed, controlled studies; } \\
\text { randomized trials with low false-positive and } \\
\text { low false-negative errors (high power) }\end{array}$ & A & $\begin{array}{l}\text { Evidence of level I or consistent findings from } \\
\text { multiple studies of level II, III or IV }\end{array}$ \\
\hline II & $\begin{array}{l}\text { At least one well-designed experimental study; } \\
\text { randomized trials with high false-positive or high } \\
\text { false-negative errors, or both (low power) }\end{array}$ & B & $\begin{array}{l}\text { Evidence of level II, III or IV, } \\
\text { and generally consistent findings }\end{array}$ \\
\hline III & $\begin{array}{l}\text { Well-designed, quasi-experimental studies such as } \\
\text { nonrandomised, controlled, single-group, } \\
\text { preoperative-postoperative comparison, } \\
\text { cohort, time or matched case-control series }\end{array}$ & $\mathrm{C}$ & $\begin{array}{l}\text { Evidence of level II, III or IV, } \\
\text { but inconsistent findings }\end{array}$ \\
\hline IV & $\begin{array}{l}\text { Well-designed, nonexperimental studies such as } \\
\text { comparative and correlational desciptive, and case studies }\end{array}$ & $\mathrm{D}$ & Little or no systematic empirical evidence \\
\hline V & Case reports and clinical examples & & \\
\hline
\end{tabular}



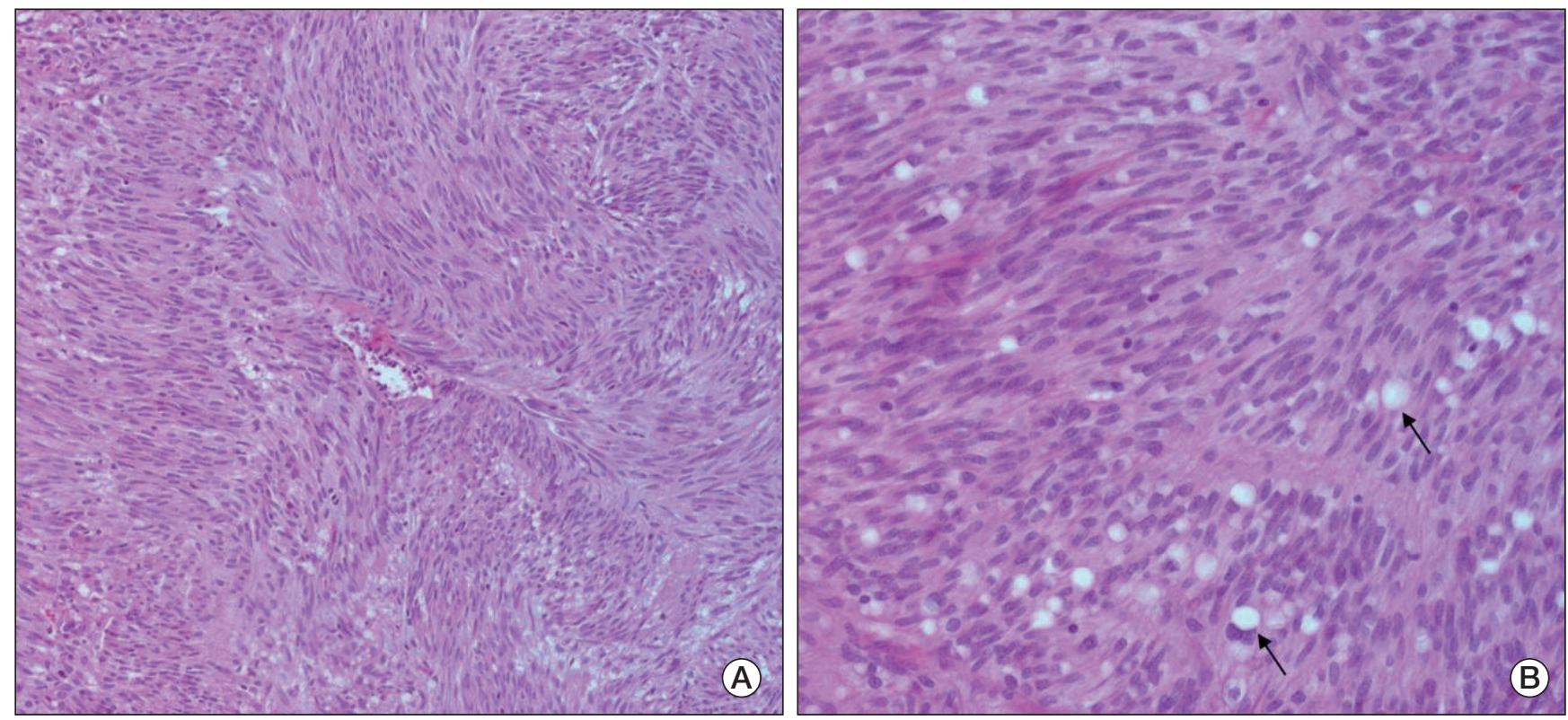

Fig. 1. Photomicrograph of a typical spindle cell type gastrointestinal stromal tumor (A, H\&E staining, $\times 200)$. In the stomach, spindle cells frequently contain many vacuoles (arrows) (B, H\&E staining, $\times 400)$.

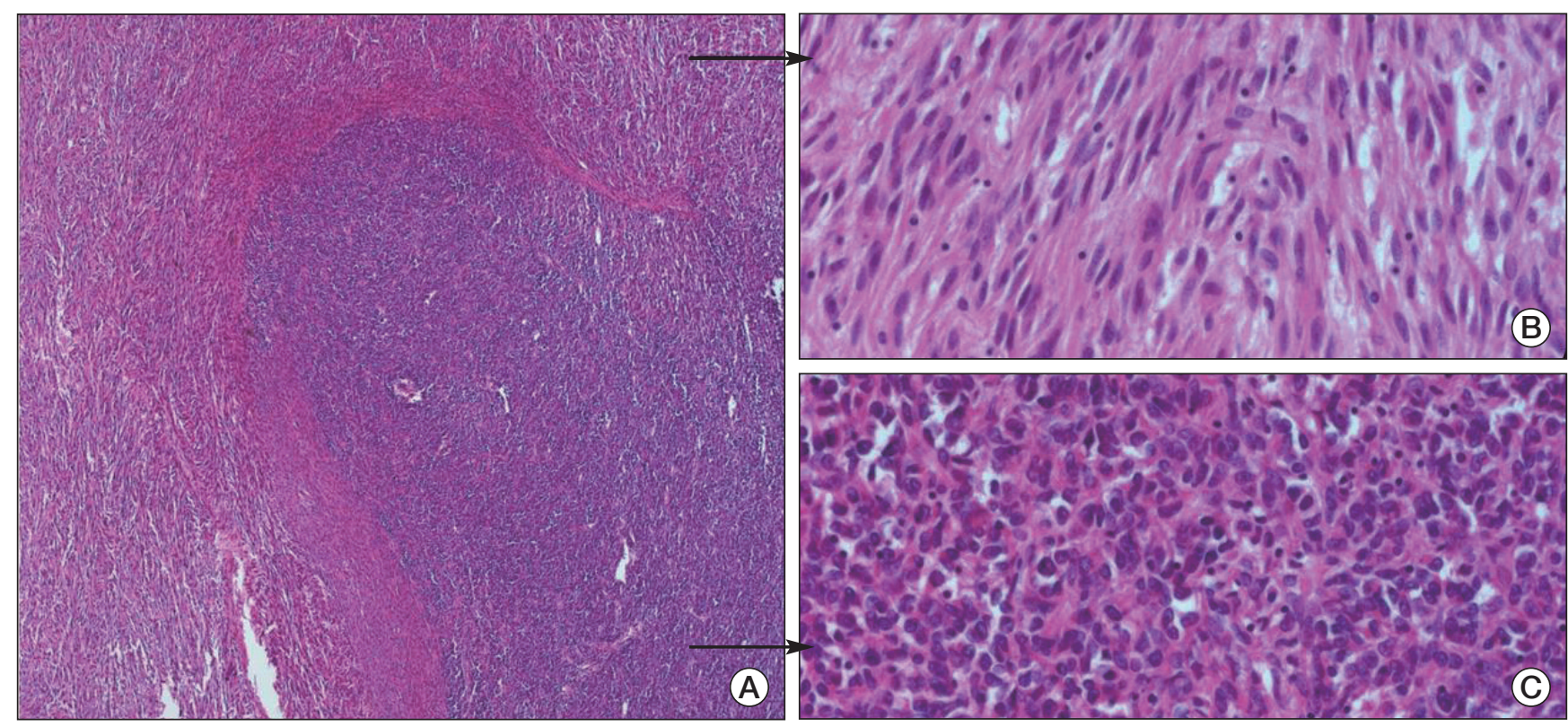

Fig. 2. In a gastrointestinal stromal tumor (GIST) of the small intestine $(A, H \& E$ staining, $\times 200)$ the malignant tumor shows typical GIST dedifferentiated histology $(\mathrm{B}, \mathrm{H} \& \mathrm{E}$ staining, $\times 400)$ and a poorly differentiated sarcoma area $(\mathrm{C}, \mathrm{H} \& \mathrm{E}$ staining, $\times 400)$.

necrosis and cystic degeneration. Microscopically, GISTs can be divided into three different histologic subgroups. Spindle cell GISTs (70\%) comprise cells with palely eosinophilic, fibrillary cytoplasm, ovoid uniform nuclei and ill-defined cell borders, often with a somewhat syncytial appearance with cells arranged in short fascicles or whorls (Fig. 1). Epithelioid GISTs (20\%) comprise rounded cells with eosinophilic to clear cytoplasm arranged in sheets and nests. The third group is characterized by mixed spindle and epithelioid cells $(10 \%)$. The frequencies of these histological types vary according to the tumor location. GISTs of the stomach typically fall into 1 of 4 spindle cell subtypes (sclerosing, palisadingvacuolated, hypercellular or sarcomatous), or 1 of 4 epitheloid subtypes (sclerosing epitheloid variant, dyscohesive epithelioid, hypercellular or sarcomatous) [15]. GISTs of the small intestine contain large amounts of skeinoid fiber and are most likely to become malignant if presenting as an epithelioid or mixed type. Rarely, malignant GISTs of the small intestine reveal a dedifferentiated histologic appearance containing typical 
GIST histology and poorly differentiated sarcoma features (Fig. 2). Many of the GISTs in the large intestine are spindle cell types. GISTs of the omentum are similar to the histological types of the stomach, whereas GISTs of the mesentery contain histological findings which are similar to the small intestine.

SDH-deficient GISTs reveal histologic clues including epithelial hypercellular features, plexiform growth pattern in the muscularis propria, and lymphovascular invasion and lymph node metastases which are otherwise rare in gastric GISTs [14].

The diagnosis of GISTs is mainly based on clinical and histological findings, but immunohistochemical staining is needed in order to confirm the diagnosis [16].
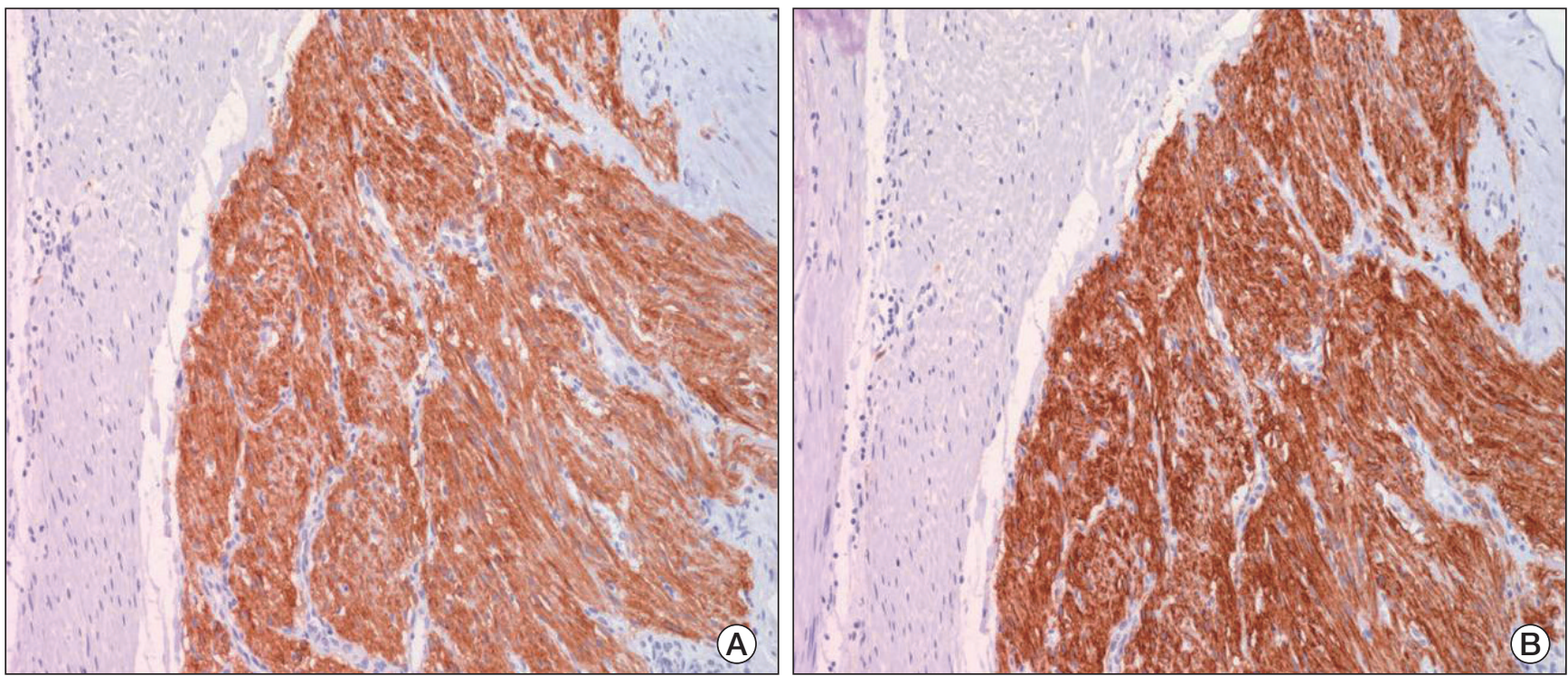

Fig. 3. Gastrointestinal stromal tumors which are positive for KIT protein (A, immunohistochemical staining, $\times 200)$ and DOG-1 (B, immunohistochemical staining, $\times 200)$. Smooth muscle bundles and blood vessels serve as internal negative controls.

Table 2. Recommended immunohistochemical markers for gastrointestinal stromal tumors (GISTs) and other immunoreactive tumors [15,16,18$23]$

\begin{tabular}{lll} 
Antibody & Positive in GISTs $(\%)$ & Other immunoreactive tumors \\
KIT $($ CD117) & $90-95$ & Melanoma, PEComa, clear cell sarcoma \\
DOG-1 & $87-98$ & Retroperitoneal leiomyoma (uterine type), peritoneal leiomyomatosis, synovial sarcomas \\
CD34 & $60-85$ of gastric GISTs & SFT, spindle cell lipoma \\
& 50 of small intestinal GISTs & PNST, vascular lesion \\
PKC-theta & 90 & PNST, smooth muscle tumor, desmoid \\
H-caldesmon & $60-80$ & Smooth muscle tumor \\
SMA & $30-40$ & Smooth muscle/myofibroblastic tumor \\
S-100 & 5 & Melanoma, PNST, granular cell tumor \\
Desmin & $1-2$ & Smooth muscle tumor \\
\hline
\end{tabular}

PEComa, perivascular epithelioid cell tumor; SFT, solitary fibrous tumor; PNST, peripheral nerve sheath tumors; PKC, protein kinase C; SMA, smooth muscle actin. 
Because KIT may also be positive for other soft tissue tumors, interpretation of KIT results based on hematoxylin and eosin findings is necessary (Table 2) [15,16,18-23].

A recently developed antibody against DOG-1 (discovered on GIST) was reported to be superior in terms of sensitivity and specificity to KIT and CD34 (Fig. 3B). The commercially available DOG-1 antibody, clone $\mathrm{K}$, is of great utility for the diagnosis of most KIT-negative GISTs, and the combination of both KIT and K9 may be used in order to accurately diagnose more than $99 \%$ of GIST cases [18]. CD34 is positive in 60 to $80 \%$ of GISTs, and the frequency of CD34 positivity depends on the tumor location. The frequency of positive CD34 is high in GISTs of the esophagus and colon (95\%), but relatively low in small bowel and extragastrointestinal sites (EGISTs). In the small intestine, CD34 is positive in $50 \%$ of cases while KIT is positive in almost $100 \%$ of cases. However, GISTs of the colon can be readily misdiagnosed as other soft tissue tumors, such as inflammatory fibroid polyp or inflammatory myofibroblastic tumor, a finding attributable to the rare occurrence of GISTs in the colon and greater incidence of KIT negative or focal staining of GISTs of the colon relative to other organs. Thus, KIT negative staining does not exclude the possibility of the occurrence of GIST, and every effort should be made to obtain the proper differential diagnosis.

Protein kinase C (PKC)-theta staining is positive in approximately $90 \%$ of GIST cases. The quality of the PKC-theta staining must be managed by observing the ganglion cells of the intermyenteric plexus as an internal positive control, and the smooth muscle or blood vessel as a negative control. When the staining is properly performed, it can serve as an important adjunct tool in the diagnosis of KIT negative GISTs, particularly those which have developed in stomach and extragastrointestinal locations [19]. H-caldesmon is positive in 60 to $80 \%$ of GISTs, a finding which may be helpful in the diagnosis of KIT-negative GISTs. Smooth muscle actin is positive in 30 to $40 \%$ of GISTs, and the frequency of the positive staining is especially high in tumors of the small bowel. S-100 and desmin are positive in $5 \%$ and 1 to $2 \%$ of GISTs, respectively.

\section{Extragastrointestinal GISTs}

In addition to the GI tract, GISTs are also found in extragastrointestinal tract, although rarely. Caution should be taken because histological and immunohistochemical findings of EGISTs are different from those of GISTs, and consequently, a correct diagnosis may be difficult.

\section{KIT-negative GISTs}

In GISTs, KIT is negative in $\sim 5 \%$ of cases. These KIT-negative GISTs are common in tumors of the stomach and extragastrointestinal locations. In such cases, examination of other immunohistochemical markers (DOG-1, PKC-theta or CD34) and mutation analyses may be useful in confirming the diagnosis. Of KIT-negative GISTs, $75 \%$ are positive for PKC-theta, 36\% are positive for DOG-1, 44\% are positive for CD34, and $40 \%$ are positive for smooth muscle actin [17,19-22].

\section{Pathologic prognostic parameters}

Morphologic risk assessment in GIST cases provides the basis for clinical management and optimal patient care. The vast majority of studies of GISTs suggest that the two most important prognostic features necessary to adequately assess the risk of aggressive behavior in a primary localized GIST are mitotic activity and tumor size. These two features were the foundations of the consensus approach for GIST risk assessment published by Fletcher et al. in 2002 [16]. Subsequent data collected by Miettinen and Lasota [24], analyzing large series of GIST cases, confirmed that tumor size and mitotic activity are essential prognostic parameters for evaluation. They also proposed a new parameter, tumor location, for use in the evaluation of the clinical behavior of localized GISTs. KGSG adopted this proposed risk stratification, but with slight modification, as there are no descriptions of locations other than the small intestine (Table 3) [15,25].

Table 3. Newly proposed risk stratification of primary localized gastrointestinal stromal tumors (GISTs) [15] with slight modifications [25]

\begin{tabular}{|c|c|c|c|c|}
\hline \multirow[b]{2}{*}{ Group } & \multicolumn{2}{|c|}{ Tumor parameters } & \multicolumn{2}{|c|}{$\begin{array}{l}\text { Patients with progressive disease during } \\
\text { follow-up and characterization of } \\
\text { malignant potential ( } \% \text { with malignant behavior) }\end{array}$} \\
\hline & Size $(\mathrm{cm})$ & Mitotic rate per 50 HPFs & Gastric GISTs & Non-gastric GISTs \\
\hline 1 & $\leq 2$ & $\leq 5$ & Very low (0) & Very low (0) \\
\hline 2 & $>2$ and $\leq 5$ & $\leq 5$ & Low (1.9) & Low (4.3) \\
\hline $3 a$ & $>5$ and $\leq 10$ & $\leq 5$ & Low (3.6) & Intermediate (24) \\
\hline $3 b$ & $>10$ & $\leq 5$ & Intermediate (12) & High (52) \\
\hline 4 & $\leq 2$ & $>5$ & $\operatorname{Low}^{\mathrm{a}}(0)$ & $\operatorname{High}^{\mathrm{a}}(50)$ \\
\hline 5 & $>2$ and $\leq 5$ & $>5$ & Intermediate (16) & High (73) \\
\hline $6 a$ & $>5$ and $\leq 10$ & $>5$ & High (55) & High (85) \\
\hline $6 b$ & $>10$ & $>5$ & High (86) & High (90) \\
\hline
\end{tabular}

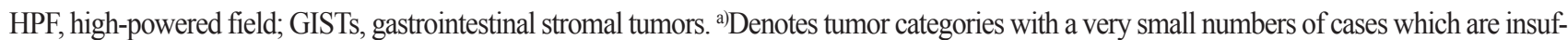
ficient for prediction of malignant potential. 


\section{Pathologic reporting for GISTs}

It has been suggested that one block per centimeter of maximum tumor dimension should be examined histologically. The pathology report should include the tumor location, size, mitosis activity (per 50 high-powered fields [HPF]), resection margin status, and the presence or absence of metastases. Discovery of metastasis or perforation during surgical operation leads to the diagnosis of a malignant GIST. A pathology report may include the tumor histological type, including the degree of cellularity and atypia, presence of necrosis or cystic changes, and any invasion into mucosa or adjacent structures. In GISTs with metastases, recurrence or rupture, diagnosis of "malignant GIST" can be made.

\section{Mutational analysis}

At present, mutation analysis is not required for the diagnosis of GISTs when the tumors have a typical histology and immunohistochemical staining pattern. However, because the presence and location of mutations in KIT or PDGFRA can have implications for prognosis and management in patients with advanced disease, mutation analysis should be considered at the time of diagnosis. Mutational analysis for KIT exons 9, 11, 13, and 17, or PDGFRA exons 12, 14, and 18 can be performed using unstained slides from tissues which are formalin-fixed, paraffin-embedded tissue, or fresh frozen.

\section{Surgical Treatment of GISTs}

\section{Surgical treatment as first-line therapy}

The primary treatment for a resectable localized GIST is surgery with the goal of complete resection without leaving residual tumor cells (R0) in the patient.

\section{Diagnosis}

Due to the difficulty in obtaining an adequate biopsy, initial diagnosis is generally made by endoscopy, endoscopic ultrasound, gastrography, or computed tomography (CT) of the abdomen. This diagnosis should be confirmed by pathologic histological findings after tumor resection. Preoperative histological diagnosis is feasible, but may be difficult to interpret definitively [26-29]. Imaging tests used in order to detect metastasis include chest X-ray (or chest CT), triphasic CT of the abdomen and pelvis, and/or magnetic resonance imaging, as necessary [30]. Positron emission tomography (PET) may be performed when evidence of metastasis may be equivocal, or for the purpose of conducting clinical trials [26].

\section{Biopsy}

There is no consensus regarding the need of endoscopic ultrasound biopsy or percutaneous biopsy for preoperative diagnosis. An important aspect of performing histological diagnosis is to not cause tumor seeding during the biopsy procedure. Therefore, unless multiple metastases are present, excisional biopsy using laparotomy is suggested [26,31]. If the differential diagnosis is unknown at the time of resection, a post-operative frozen tissue examination must be performed in order to elucidate the treatment strategy for the GIST, as the treatment strategy varies for an adenocarcinoma vs. a lymphoma. Biopsy is necessary when planning neoadjuvant therapy.

\section{Indications for surgery}

Due to the high potential for malignancy associated with cases of GIST, resection should be the first-line treatment [26,32]. We strongly recommend resection for tumors larger than $2 \mathrm{~cm}$ or those that are growing [26]. Smaller tumors $(<2 \mathrm{~cm})$ confer a lower potential for malignancy and they may also be observed. However, a small tumor size does not exclude the potential for malignant transformation. Therefore, patients should be informed about the possibility of malignancy, even if only small tumors are detected.

\section{Surgical margins}

The main objectives of surgical treatment are to acquire negative margins and to resect the tumor without causing tumor rupture. In the case of inadvertent tumor infiltration into surrounding organs, regardless of tumor size, a complete en bloc resection with negative margins should be performed [26,31,32]. Even if the tumors are small, an endoscopic shellout procedure or enucleation should be avoided if GIST is suspected. In many cases, wedge resection of gastric GISTs and segmental resection of small bowel GISTs are appropriate treatments. Based on the tumor size and location, subtotal or total gastrectomy may be performed. We recommend en bloc resection for omental or mesenteric GISTs. Adjacent organs which adhere to the tumor should also be completely resected en bloc to avoid tumor rupture or intra-abdominal seeding [31].

\section{Laparoscopic resection}

Laparoscopic resection is a feasible surgical approach if intra-abdominal tumor rupture or seeding is unlikely. Laparoscopic resection should follow the principles of oncologic surgery. Generally, it is reserved for small, favorably located gastric GISTs [33-36]. Intra-operative endoscopy or laparoscopic ultrasound may be used to assist the laparoscopic procedure as needed. 


\section{Lymphadenectomy}

Unlike adenocarcinoma, GISTs rarely metastasize to local or regional lymph nodes. Therefore, lymphadenectomy is warranted only if metastasis is suspected, i.e., the observation of enlarged lymph nodes.

\section{Post-operative follow-up and surveillance}

For patients in high- or intermediate-risk groups we recommend post-operative surveillance of the abdomen and pelvis by the use of a CT scan every 3 to 4 months for the first 3 years after surgery, every 6 months until 5 years after surgery, and an annual assessment thereafter [26,31]. For patients in the low or very low risk groups, we recommend a followup CT scan every 6 months for the 5 years after surgery. Ultrasonography may replace the use of CT once each year [26,31]. The use of PET for the purpose of post-operative surveillance has not been appropriately established, and clinical studies of its use are ongoing. Most recurrences occur within 2 years after surgery with the liver and peritoneum being the most common sites [37]. Due to the high incidence of gastric cancer in Korea, the National Cancer Screening program recommends biennial stomach cancer screening by endoscopy or by the use of the upper GI series for men and women older than 40 years of age.

\section{Post-imatinib resection in metastatic GISTs}

Medical treatment by imatinib alone usually does not result in complete response in cases of metastatic GIST. In addition, it is unusual for positive responses to be maintained indefinitely. It is well recognized that, after initiation of treatment with imatinib, clones of resistant tumor cells develop continuously over time. Therefore, in cases of partial response (PR) or stable disease (SD) which occur after an adequate duration of imatinib therapy (usually 4 to 12 months), complete surgical resection of the residual tumor may be considered in order to reduce the risk of development of resistant clones. Several retrospective studies have suggested that resection of residual lesions could prolong progression-free survival (PFS) if the surgery is performed while the tumors are under the control of imatinib (either in PR or SD) treatment [38,39]. It is emphasized that imatinib therapy should continue after resection [26]. However, the efficacy of resection of residual tumors after imatinib therapy has not been established, and several Phase III clinical trials investigating the role of surgical resection in this setting are either planned or are ongoing worldwide. Hepatic or peritoneal metastases may be locally treated using radiofrequency ablation or chemoembolization. Importantly, management of GIST should be coordinated by a multidisciplinary team of experienced medical and surgical specialists.

\section{Medical Treatment of GISTs}

\section{Adjuvant treatment}

Adjuvant treatment with imatinib is provided in order to enhance the possibility of curing the cancer through the eradication of microscopic lesions that may remain after the complete resection of visual tumors. Given the substantial efficacy that imatinib provides for metastatic or recurrent GISTs, it appears to possess significant potential as an adjuvant treatment.

In the American College of Surgeons Oncology Group Z9001 study of patients with tumor diameter $\geq 3 \mathrm{~cm}$ who received imatinib for one year following complete surgical resection, imatinib demonstrated a significant increase in recurrence-free survival (RFS) as compared with a placebo, although improvement in overall survival (OS) was not observed [40]. The benefit in RFS appeared to be related to tumor size with the most marked improvement observed in patients who had a large tumor $(\geq 10 \mathrm{~cm})$.

A Korean Phase II study recently presented in an abstract, evaluated the efficacy of 2-years of imatinib adjuvant therapy in patients who were at high risk of recurrence (tumor size $\geq 5 \mathrm{~cm}$ and mitotic index $\geq 5 / 50$ HPF, tumor size $\geq 10 \mathrm{~cm}$, or mitotic index $\geq 10 / 50 \mathrm{HPF}$ ) based on the US National Institutes of Health $(\mathrm{NIH})$ risk criteria, and who had the KIT exon 11 mutation which has been recognized as an independent risk factor for recurrence in a Korean retrospective study [41,42]. The RFS for these patients appeared to be greatly improved as compared to historical data.

Results from the recently completed randomized controlled SSGXVIII/AIO trial suggested that adjuvant imatinib administered for 36 months improved RFS and OS, as compared to adjuvant imatinib administered for 12 months, for patients with a high estimated risk of recurrence after surgery [43]. In this trial, high risk for GIST recurrence was defined as: tumor diameter $>5 \mathrm{~cm}$ and mitotic count $>5 / 50 \mathrm{HPF}$, tumor diameter $>10 \mathrm{~cm}$, any size tumor with a mitotic count $>10 / 50$ HPF, spontaneous tumor rupture into the peritoneal cavity, or tumor rupture at surgery. Patients were randomized into adjuvant imatinib treatment groups of 12 months $(\mathrm{n}=200)$ and 36 months $(\mathrm{n}=200)$. At a median follow-up duration of 54 months, a statistically significant difference in RFS was observed between the two treatment arms $(p<0.0001)$ of the intention-to-treat population $(\mathrm{n}=397)$. The RFS probability estimate at 5 years was $47.9 \%$ in the 12 -month arm and $65.6 \%$ in the 36-month arm. The hazard ratio (HR) for RFS between the treatment arms was $0.46(95 \%$ confidence interval [CI], 0.32 to 0.65 ), resulting in a $54 \%$ reduction in the risk of recurrence in favor of the 36-month arm. Similar results were obtained in the efficacy population ( $\mathrm{n}=358$ ): the RFS probability estimate at 5 years was $50.3 \%$ in the 12 -month arm and $67.4 \%$ in the $36-$ month arm, and the HR for RFS between treatment arms was 0.46 (95\% CI, 0.31 to $0.68 ; \mathrm{p}<0.0001$ ).

In addition, there was a statistically significant difference in OS observed between the treatment arms $(\mathrm{p}=0.019)$. The estimated 5-year OS was $81.7 \%$ in the 12 -month arm and $92 \%$ in the $36-$ month arm. The HR for the OS between treatment arms was 0.45 (95\% CI, 0.22 to 0.89 ), 
resulting in a 55\% reduction in the risk of death in favor of the 36-month arm. Safety was assessed in 392 patients. Ninety-two patients discontinued therapy: $29(15 \%)$ in the 12-month arm and $63(32 \%)$ in the 36-month arm. The majority of those that discontinued therapy did so due to adverse events. There were a total of $37(9.4 \%)$ deaths during the study: 25 in the 12-month arm and 12 in the 36-month arm. There were 14 and 7 GISTrelated deaths in the 12-month and 36-month arms, respectively. Based on the results of the SSGXVIII/AIO trial, NCCN updated their guideline for GIST to recommend at least 36 months of post-operative treatment with imatinib for patients with high-risk GISTs (tumor greater than $5 \mathrm{~cm}$ in size with high mitotic rate $>5$ mitoses $/ 50 \mathrm{HPF}$, or a risk of recurrence of greater than $50 \%$ ) [1].

In the $2002 \mathrm{NIH}$ consensus risk classification, only tumor size and mitotic count were included. More recent risk classifications incorporated primary tumor site and/or tumor rupture data in addition to tumor size and mitotic count in the assessment. The current expert consensus is to recommend adjuvant imatinib for patients at high risk of relapse, but not for those at low relapse risk. However, there has been no consensus for patients at intermediate risk $[24,44]$. Patients with tumor rupture should be considered as having a high likelihood of micrometastasis and they should be treated with imatinib either in adjuvant setting or in palliative setting. In addition to the risk assessment, mutational analysis may be helpful for the selection of patients unlikely to benefit from adjuvant imatinib treatment. For example, GIST patients with the D842V mutation in PDGFRA exon 18 are known to be unresponsive to imatinib. With the currently available data, it is recommended to use imatinib as an adjuvant treatment for at least 36 months for patients with high-risk GISTs [II, C]. However, an optimal duration of adjuvant imatinib treatment has yet to be determined. Other ongoing adjuvant studies (e.g. the EORTC 62024 and PERSIST-5 trials) may help answer to this question.

\section{Neoadjuvant treatment}

Outside of a clinical trial, neoadjuvant treatment with imatinib is not recommended unless there is clinically significant justification for reducing the tumors sizes in order to improve surgery results [26]. Neoadjuvant treatment with imatinib may also be considered, if $\mathrm{R} 0$ resection is infeasible, for the purpose of preserving organ function in patients with GISTs of the rectum, esophagus or duodenum, or for avoiding total gastrectomy of gastric GIST which is huge or located close to esophagogastric junction [45-47]. When such neoadjuvant treatment is considered, progression and response of tumors, both before and during the treatment, should be assessed very carefully by $\mathrm{CT}$ and/or PET scan by an experienced multidisciplinary team. Early assessment of tumor response by $\mathrm{CT}$ and/or PET is recommended such that surgery is not delayed in cases of non-responding tumors. Duration of neoadjuvant imatinib therapy may vary according to treatment response, and surgery should be performed after sufficient shrinkage of the tumors is observed (typically from 4 to 6 up to 12 months after initiation of imatinib treatment) [26]. Mutational analysis may be helpful in order to exclude GIST patients who will not respond to imatinib treatment.

\section{Advanced disease}

\section{1) Initiation of imatinib}

Once an advanced GIST is diagnosed, imatinib should be immediately initiated regardless of the presence or absence of symptoms. Surgical resection cannot replace the imatinib therapy for the treatment of metastatic tumor. Imatinib therapy should be continued even after resection of limited metastasis to liver or peritoneum because cure is hard to achieve, even if the tumors are completely resected visually and histologically [37,48]. Adjuvant therapy does not apply in these cases.

\section{2) Optimal imatinib dosage}

The optimal initial dose of imatinib is $400 \mathrm{mg}$ per day. In a large European Phase III study that compared 400 to $800 \mathrm{mg}$ daily doses, the 800 $\mathrm{mg}$ did not achieve an increased survival rate yet the side-effects did increase $[49,50]$. However, in the subgroup analysis, a clinically significant improvement for PFS was observed in the $800 \mathrm{mg}$ group which possessed KIT exon 9 mutations. Thus, high-dose imatinib is now recommended in Western countries as the initial treatment for patients with KIT exon 9-mutant GISTs [51]. However, no large prospective studies have compared the clinical benefit of imatinib treatment for different GIST genotypes in Asian patients $[42,52,53]$. Recently reported results from a large-scale retrospective analysis (the KGSG study) suggested that Korean patients with KIT exon 9 mutations would also benefit from treatment with high-dose imatinib [54]. In this study, clinical data was collected from 370 consecutive patients with locally advanced, unresectable, metastatic, or recurrent GISTs who were treated with imatinib $400 \mathrm{mg} /$ day between August 2001 and December 2007 at 20 Korean institutions. Tumor genotypes were determined for 290 patients by direct DNA sequencing of their KIT exons 9, 11, 13 and 17, and of PDGFRA exons 12, 14 and 18. Of the patients assessed for each genotype, $261(90.0 \%)$ had KIT mutations: 222 (76.6\%) in exons 11, and $35(12.1 \%)$ in exons 9, and 2 each (0.7\%) in exons 13 and 17. Four patients (1.4\%) had mutations in the PDGFRA gene: 1 in exon 12 and 3 in exon 18. Twenty-five patients (8.6\%) had no detectable mutations. The best responses from the 235 patients with measurable lesions were: 15 complete responses (6.4\%), 126 with PRs (53.5\%), 86 with SDs (36.6\%) and 8 with progressive diseases (3.4\%). Compared with the patients with KIT exon 11 mutations, those with KIT exon 9 mutations had a lower objective response rate $(63.6 \%$ vs. $36.7 \%$; $\mathrm{p}=0.007)$ and a shorter PFS (median, 49.4 months vs. 28.7 months; $p=0.001$ ), although no statistical difference in OS was observed between these genotypes. The KGSG study suggested that high-dose imatinib should be considered in Korean patients with KIT exon 9 mutations, just as recommended for Western patients with the similar genotype [IV, C]. However, this conclusion needs to be rigorously tested. As a result, a Phase II study has been initiated in order to assess the feasibility of imatinib dose escalation, as well as determine the safety and efficacy of imatinib as a first-line treatment at an escalated dose in Korean patients with metastatic or unresectable GISTs who harbor KIT exon 9 mutations. 


\section{3) Duration of imatinib treatment and surgical resection of respond- ing tumors}

Treatment with imatinib should be continued indefinitely unless the disease progresses, intolerable adverse events occur, or unless the patient refuses treatment. If imatinib treatment is discontinued after positive tumor response is achieved, the disease continues to progress in most cases. If the disease progresses following imatinib discontinuation, many patients respond to the reintroduction of imatinib but the tumor response may be weaker than the gains achieved prior to treatment interruption [55]. Therefore, imatinib treatment should not be interrupted outside of a clinical trial unless the interruption is clinically indicated. Surgical resection of stabilized tumor lesions can be considered after discussion with the patient using a multidisciplinary approach.

\section{4) Standard tests for tumor response}

To date, CT has been the most useful diagnostic tool used for determining response to treatment. We recommend dynamic or triphasic CT scanning through arteries and veins after contrast enhancement [30]. Fluorodeoxyglucose (FDG) PET is highly sensitive in early tumor response, but given its cost and limited availability, it is not easy to include in basic imaging testing [56-58]. Intervals used to determine response may vary according to the particular clinical situation, but tumor response is usually determined every 3 to 4 months after the initial response is confirmed.

\section{5) Criteria for determination of response}

Despite the therapeutic effects observed at the beginning of treatment, judgment regarding the tumor response or determination of treatment continuation, based solely on tumor size, should be avoided as tumors may enlarge due to intratumoral hemorrhage or myxoid degeneration [30,59-61]. Because GIST is a hypervascular neoplasm which exhibits hypoattenuating findings resulting from reduced vascularity, hyaline degeneration and occasional cystic changes following imatinib treatment, caution is required in the application of Response Evaluation Criteria in Solid Tumors (RECIST) or World Health Organization (WHO) criteria. The development of new criteria is warranted, particularly for liver metastasis, as tumor response is determined solely using the portal-venous phase CT where small and new lesions with hypodensity may be seen; the clear margins of these lesions are most likely secondary to the necrosis of preexisting tumors that were present prior to treatment and had the same radiodensity as the hepatic parenchyma, a finding requiring differential diagnosis [30,61]. Both tumor size and density should be considered for the response evaluation. Improvement of patient symptoms, reduction in degree of CT attenuation coefficient (Hounsefield units), or change in maximum standardized uptake value (SUVmax) upon PET scan may also be used in order to determine tumor response [30,59-61].

\section{6) Recurrence and progression}

Recurrence or progression may include the appearance of a new lesion at the surgery site, development of a new metastasis, or an increase in an existing tumor's size. In some cases, a new intratumoral nodule or increased solid tissue formation in a hypodense tumor which previously responded to treatment is identified as a recurrence or progression. The patterns of the progression cannot be determined based on RECIST or WHO criteria, and thus, the interior and cell walls of each lesion should be carefully examined $[30,62]$.

\section{7) Treatment of disease progression during imatinib therapy}

Resistance is classified into primary resistance and secondary resistance. Primary resistance is defined as progression within the first 6 months of imatinib therapy, with most cases progressing multifocally [26]. Secondary resistance is defined as progression after 6 months of the initiation of imatinib therapy, and generally two types of progression are seen $[26,30]$ as described below.

\section{(1) Focal progression}

Focal progression occurs when one lesion, or a limited number of multiple lesions, exhibit intratumoral nodules or increase in size which results in increased FDG uptake on a PET scan while the remainder of lesions remain relatively well controlled. Treatment for focal progression requires multidisciplinary approaches. Local treatments such as resection of localized metastasis to the liver or peritoneum, radiofrequency ablation, as well as chemoembolization can be considered in order to control focally progressing lesions. However, systemic treatment should also be continued in order to control any hidden micrometastatic lesions. If locally progressing lesions are completely removed, the standard dose of imatinib can be continued. If those lesions were not completely removed, imatinib dose escalation or a switch to sunitinib should be considered. No prospective studies on the efficacy of local treatment for focal progression have been conducted. Studies have suggested that some patients with focal progression may benefit from local treatment, but others have shown general progression within a short time after local treatment $[38,39,63]$.

\section{(2) General progression (multifocal resistance)}

General progression is characterized by a majority of multiple lesions progressing simultaneously. The efficacy of local treatment on multifocal resistance is extremely limited and mostly negative [38]. Therefore, local treatment for general progression is not recommended except for palliation of symptoms. Administration of imatinib at increased doses, or a second-line drug such as sunitinib, should be considered.

\section{(3) Increase of imatinib dose at disease progression}

When the disease progresses during treatment using the standard imatinib dose of $400 \mathrm{mg}$ per day, an increased dose of $800 \mathrm{mg}$ per day has been shown to induce objective response or to stabilize tumors for a limited period in approximately 30 to $40 \%$ of patients [48,64]. With a daily dose of $800 \mathrm{mg}$, other than malaise and anemia, adverse events associated with imatinib have not been found to increase. If an intolerable side-effect occurs, the imatinib dose may be reduced to $600 \mathrm{mg}$ per day [64]. When severe adverse events are expected with a direct dose escalation to $800 \mathrm{mg}$ per day, imatinib can be first escalated to $600 \mathrm{mg}$ per day, subsequently to $800 \mathrm{mg}$ per day. The median PFS is 
approximately 3 months and the 12 -month PFS rate is 18 to $30 \%$ with imatinib dose escalation [65].

\section{(4) Use of sunitinib at disease progression}

Sunitinib is a new tyrosine kinase inhibitor that inhibits the vascular endothelial growth factor receptor as well as KIT and PDGFRA. As a result, sunitinib may inhibit tumor growth by both direct antitumor activity and antiangiogenic activity [66]. Sunitinib has been approved for the treatment of patients with advanced GISTs after failure of firstline imatinib. Results from a Phase III pivotal study showed that sunitinib at a daily dose of $50 \mathrm{mg}$ with a 4 weeks on and 2 weeks off schedule was significantly superior in terms of time to progression over a placebo (median, 27.3 weeks vs. 6.4 weeks; $p<0.0001$ ) [66]. Because progression during the rest period was occasionally observed, a continuous dosing schedule with a lower daily dose (37.5 mg per day) was also developed and proven to be effective and well tolerated. However, no randomized trials have been performed in order to compare the intermittent and the continuous dosing schedules. Therefore, a continuous dosing schedule with a lower daily dose of sunitinib should only be considered as an option in unique cases.

\section{(5) Use of conventional cytotoxic chemotherapy at disease progres-} sion

No conventional cytotoxic chemotherapy has ever been reported to be effective in GISTs. Thus, we do not recommend the use of these drugs except in clinical studies [26].

\section{(6) Continuous use of imatinib or sunitinib after previous failure} of both treatments

There is no effective systemic treatment option after failure of both imatinib and sunitinib. General oncology principles indicate that the use of the same agent that has already failed is neither beneficial nor recommended. However, in spite of resistance to these agents in a majority of tumor cells in this scenario, at least a fraction of the tumor cells may remain responsive to the agents. Therefore, it is permitted and recommended by many national regulatory agencies to continue the use of one of these agents in order to slow the progression of the disease, even after the tumor is determined to be resistant according to RECIST. For this indication, imatinib at a daily dose of $400 \mathrm{mg}$ may be preferred to the use of sunitinib. However, a well-designed clinical trial for both agents is needed in order to evaluate the effectiveness of continuous treatment using imatinib or sunitinib after disease progression.

\section{Conclusion}

This is the third version of the original guideline published in 2007 and updated in 2010 by KGSG for the diagnosis and treatment of GISTs, as appropriate for Korean patients. Through a series of workshops conducted in order to review and discuss the evolving new evidence, we have updated the clinical recommendations to reflect recent changes in the diagnosis and surgical and medical treatment of GISTs. We expect this guideline will enhance the accuracy of diagnoses performed by members of the Korean association of physicians involved in GIST patient care, resulting in optimal efficacy of treatment.

\section{Conflicts of Interest}

Dr. Yoon-Koo Kang has received honoraria for lectures from Novartis and Pfizer.

\section{References}

1. National Comprehensive Cancer Network. NCCN clinical practice guidelines in oncology: soft tissue sarcoma. Version 2.2011 [Internet]. Fort Washington, PA: National Comprehensive Cancer Network; 2011 [cited 2012 Mar 2]. Available from: http://www.nccn.org/professionals/ physician_gls/pdt/sarcoma.pdf.

2. Casali PG, Blay JY; ESMO/CONTICANET/EUROBONET Consensus Panel of Experts. Gastrointestinal stromal tumours: ESMO clinical practice guidelines for diagnosis, treatment and follow-up. Ann Oncol. 2010;21 Suppl 5:v98-102.

3. Nishida T, Hirota S, Yanagisawa A, Sugino Y, Minami M, Yamamura Y, et al. Clinical practice guidelines for gastrointestinal stromal tumor (GIST) in Japan: English version. Int J Clin Oncol. 2008:13:416-30

4. Demetri GD, Antonia S, Benjamin RS, Bui MM, Casper ES, Conrad EU III, et al. National Comprehensive Cancer Network Practice Guidelines in Oncology Soft Tissue Sarcoma. J Natl Compr Canc Netw internet. Fort Washington, PA: National Comprehensive Cancer Network; 2011 [cited 2012 Mar 2]. Available from: http://www.nccn.org/professionals/ physician_gls/ f_quidelines.asp

5. Korean Gastrointestinal Stromal Tumor Study Group; Kang YK. Clinical practice guideline for adequate diagnosis and effective treatment of gastrointestinal stromal tumor in Korea. J Korean Med Assoc. 2007;50:830-41.

6. Kang YK, Kim KM, Sohn T, Choi D, Kang HJ, Ryu MH, et al. Clinical practice guideline for accurate diagnosis and effective treatment of gastrointestinal stromal tumor in Korea. J Korean Med Sci. 2010;25:1543-52.

7. Nelson H, Petrelli N, Carlin A, Couture J, Fleshman J, Guillem J, et al. Guidelines 2000 for colon and rectal cancer surgery. J Natl Cancer Inst. 2001:93:583-96.

8. Kang DY, Park CK, Choi JS, Jin SY, Kim HJ, Joo M, et al. Multiple gastrointestinal stromal tumors: clinicopathologic and genetic analysis of 12 patients. Am J Surg Pathol. 2007; 31:224-32.

9. Rubin BP. Heinrich MC, Corless CL. Gastrointestinal stromal tumour. Lancet. 2007:369:173141.

10. Kim KM, Kang DW, Moon WS, Park JB, Park CK, Sohn JH, et al. Gastrointestinal stromal tumors in Koreans: its incidence and the clinical, pathologic and immunohistochemical findings. J Korean Med Sci. 2005:20:977-84.

11. Cho MY, Sohn JH, Kim JM, Kim KM, Park YS, Kim WH, et al. Current trends in the epidemiological and pathological characteristics of gastrointestinal stromal tumors in Korea, 20032004. J Korean Med Sci. 2010;25:853-62.

12. Demetri GD, Benjamin RS, Blanke CD, Blay JY, Casali P, Choi H, et al. NCCN Task Force report: management of patients with gastrointestinal stromal tumor (GIST) — update of the NCCN clinical practice guidelines. J Natl Compr Canc Netw. 2007;5 Suppl 2:S1-29.

13. Heinrich MC, Corless CL, Demetri GD, Blanke CD, von Mehren M, Joensuu H, et al. Kinase 
mutations and imatinib response in patients with metastatic gastrointestinal stromal tumor. J Clin Oncol. 2003;21:4342-9.

14. Miettinen M, Wang ZF, Sarlomo-Rikala M, Osuch C, Rutkowski P, Lasota J. Succinate dehydrogenase-deficient GISTs: a clinicopathologic, immunohistochemical, and molecular genetic study of 66 gastric GISTs with predilection to young age. Am J Surg Pathol. 2011; 35:1712-21.

15. Miettinen M, Lasota J. Gastrointestinal stromal tumors: review on morphology, molecular pathology, prognosis, and differential diagnosis. Arch Pathol Lab Med. 2006;130:1466-78.

16. Fletcher CD, Berman JJ, Corless C, Gorstein F, Lasota J, Longley BJ, et al. Diagnosis of gastrointestinal stromal tumors: a consensus approach. Hum Pathol. 2002;33:459-65.

17. Liegl B, Hornick JL, Lazar AJ. Contemporary pathology of gastrointestinal stromal tumors. Hematol Oncol Clin North Am. 2009;23:49-68.

18. Kang GH, Srivastava A, Kim YE, Park HJ, Park CK, Sohn TS, et al. DOG-1 and PKC- $\theta$ are useful in the diagnosis of KIT-negative gastrointestinal stromal tumors. Mod Pathol. 2011;24,866-75.

19. Kim KM, Kang DW, Moon WS, Park JB, Park CK, Sohn JH, et al. PKCtheta expression in gastrointestinal stromal tumor. Mod Pathol. 2006;19:1480-6.

20. Lee HE, Kim MA, Lee HS, Lee BL, Kim WH. Characteristics of KIT-negative gastrointestinal stromal tumours and diagnostic utility of protein kinase $\mathrm{C}$ theta immunostaining. J Clin Pathol. 2008:61:722-9.

21. Orosz Z, Tornóczky T, Sápi Z. Gastrointestinal stromal tumors: a clinicopathologic and immunohistochemical study of 136 cases. Pathol Oncol Res. 2005;11:11-21.

22. Medeiros F, Corless CL, Duensing A, Hornick JL, Oliveira AM, Heinrich MC, et al. KIT-negative gastrointestinal stromal tumors: proof of concept and therapeutic implications. Am J Surg Pathol. 2004;28:889-94.

23. Miettinen M, Wang ZF, Lasota J. DOG-1 antibody in the differential diagnosis of gastrointestinal stromal tumors: a study of 1840 cases. Am J Surg Pathol. 2009;33:1401-8.

24. Miettinen M, Lasota J. Gastrointestinal stromal tumors: pathology and prognosis at different sites. Semin Diagn Pathol. 2006;23:70-83.

25. Miettinen M, Sarlomo-Rikala M, Lasota J. Gastrointestinal stromal tumors: recent advances in understanding of their biology. Hum Pathol. 1999;30:1213-20.

26. Blay JY, Bonvalot S, Casali P, Choi H, Debiec-Richter M, Dei Tos AP, et al. Consensus meeting for the management of gastrointestinal stromal tumors. Report of the GIST Consensus Conference of 20-21 March 2004, under the auspices of ESMO. Ann Oncol. 2005;16:56678.

27. Brunicardi FC, Andersen DK, Billiar TR, Dunn DL, Hunter JG, Pollock RE. Schwartz's principles of surgery. 8th ed. New York: McGraw-Hill; 2005.

28. Townsend CM Jr, Sabiston DC. Sabiston textbook of surgery: the biological basis of modern surgical practice. 17th ed. Philadelphia: W.B. Saunders Co., 2004.

29. Trent JC, Benjamin RS. New developments in gastrointestinal stromal tumor. Curr Opin Oncol. 2006;18:386-95

30. Ryu MH, Lee JL, Chang HM, Kim TW, Kang HJ, Sohn HJ, et al. Patterns of progression in gastrointestinal stromal tumor treated with imatinib mesylate. Jpn J Clin Oncol. 2006;36:1724.

31. Gold JS, Dematteo RP. Combined surgical and molecular therapy: the gastrointestinal stromal tumor model. Ann Surg. 2006;244:176-84.

32. Iwahashi M, Takifuji K, Ojima T, Nakamura M, Nakamori M, Nakatani Y, et al. Surgical management of small gastrointestinal stromal tumors of the stomach. World J Surg. 2006;30:2835.

33. Choi SM, Kim MC, Jung GJ, Kim HH, Kwon HC, Choi SR, etal. Laparoscopic wedge resection for gastric GIST: Iong-term follow-up results. Eur J Surg Oncol. 2007;33:444-7.

34. Novitsky YW, Kercher KW, Sing RF, Heniford BT. Long-term outcomes of laparoscopic resection of gastric gastrointestinal stromal tumors. Ann Surg. 2006;243:738-45.

35. Otani Y, Furukawa T, Yoshida M, Saikawa Y, Wada N, Ueda M, et al. Operative indications for relatively small $(2-5 \mathrm{~cm})$ gastrointestinal stromal tumor of the stomach based on analysis of 60 operated cases. Surgery. 2006;139:484-92.

36. Pister PW, Blay JY. GIST guidelines: from evidence to practice, evolving consensus in GIST management meeting monograph. In: GIST Global Opinion Leader Summit; 2007 Oct 4-8; Geneva, Switzerland.

37. DeMatteo RP, Lewis JJ, Leung D, Mudan SS, Woodruff JM, Brennan MF. Two hundred gastrointestinal stromal tumors: recurrence patterns and prognostic factors for survival. Ann Surg. 2000;231:51-8.

38. Raut CP, Posner M, Desai J, Morgan JA, George S, Zahrieh D, et al. Surgical management of advanced gastrointestinal stromal tumors after treatment with targeted systemic therapy using kinase inhibitors. J Clin Oncol. 2006;24:2325-31.

39. Sym SJ, Ryu MH, Lee JL, Chang HM, Kim TW, Kim HC, et al. Surgical intervention following imatinib treatment in patients with advanced gastrointestinal stromal tumors (GISTs). J Surg Oncol. 2008:98:27-33.
40. DeMatteo RP, Ballman KV, Antonescu CR, Maki RG, Pisters PW, Demetri GD, et al. Adjuvant imatinib mesylate after resection of localised, primary gastrointestinal stromal tumour: a randomised, double-blind, placebo-controlled trial. Lancet. 2009;373:1097-104.

41. Kang B, Lee J, Ryu M, Im S, Park S, Kang W, et al. A phase II study of imatinib mesylate as adjuvant treatment for curatively resected high-risk localized gastrointestinal stromal tumors. J Clin Oncol. 2009;27(S):e21515.

42. Kim TW, Ryu MH, Lee H, Sym SJ, Lee JL, Chang HM, et al. Kinase mutations and efficacy of imatinib in Korean patients with advanced gastrointestinal stromal tumors. Oncologist. 2009;14:540-7.

43. Joensuu H, Eriksson M, Hatrmann J, Sundby Hall K, Schutte J, Reichardt A, et al. Twelve versus 36 months of adjuvant imatinib (IM) as treatment of operable GIST with a high risk of recurrence: final results of a randomized trial (SSGXVIII/AIO). J Clin Oncol. 2011;29(S):LBA1.

44. Joensuu H. Risk stratification of patients diagnosed with gastrointestinal stromal tumor. Hum Pathol. 2008;39:1411-9.

45. Bumming P, Andersson J, Meis-Kindblom JM, Klingenstierna H, Engström K, Stierner U, et al. Neoadjuvant, adjuvant and palliative treatment of gastrointestinal stromal tumours (GIST) with imatinib: a centre-based study of 17 patients. Br J Cancer. 2003;89:460-4.

46. Loughrey MB, Mitchell C, Mann GB, Michael M, Waring PM. Gastrointestinal stromal tumour treated with neoadjuvant imatinib. J Clin Pathol. 2005;58:779-81.

47. Oh JS, Lee JL, Kim MJ, Ryu MH, Chang HM, Kim TW, et al. Neoadjuvant imatinib in locally advanced gastrointestinal stromal tumors of stomach: report of three cases. Cancer Res Treat. 2006;38:178-83.

48. DeMatteo RP, Heinrich MC, El-Rifai WM, Demetri G. Clinical management of gastrointestinal stromal tumors: before and after STI-571. Hum Pathol. 2002;33:466-77.

49. Rankin C, von Mehren M, Blanke C, Benjamin R, Fletcher CDM, Bramwell V, et al. Dose effect of imatinib (im) in patients (pts) with metastatic GIST - Phase III Sarcoma Group Study S0033. J Clin Oncol. 2004;22(14S):9005.

50. Verweij J, Casali PG, Zalcberg J, LeCesne A, Reichardt P, Blay JY, et al. Progression-free survival in gastrointestinal stromal tumours with high-dose imatinib: randomised trial. Lancet. 2004:364:1127-34.

51. Debiec-Rychter M, Sciot R, Le Cesne A, Schlemmer M, Hohenberger P, van Oosterom AT, et al. KIT mutations and dose selection for imatinib in patients with advanced gastrointestinal stromal tumours. Eur J Cancer. 2006;42:1093-103.

52. Du CY, Shi YQ, Zhou Y, Fu H, Zhao G. The analysis of status and clinical implication of KIT and PDGFRA mutations in gastrointestinal stromal tumor (GIST). J Surg Oncol. 2008;98:1758.

53. Yeh CN, Chen TW, Lee HL, Liu YY, Chao TC, Hwang TL, et al. Kinase mutations and imatinib mesylate response for 64 Taiwanese with advanced GIST: preliminary experience from Chang Gung Memorial Hospital. Ann Surg Oncol. 2007;14:1123-8.

54. Kang HJ, Ryu MH, Kim KM, Park YS, Choi J, Ryoo BY, et al. Imatinib efficacy by tumor genotype in Korean patients with advanced gastrointestinal stromal tumors (GIST): The Korean GIST Study Group (KGSG) study. Acta Oncol. 2012;51:528-36.

55. Domont J, Blay J, Ray-Coquard IL, Bui Nguyen B, Adenis A, Rios M, et al. Influence of imatinib interruption and imatinib rechallenge on the residual tumor volume in patients with advanced GIST: results of the BFR14 prospective French Sarcoma Group randomized phase III trial. J Clin Oncol. 2011;29(S):10054.

56. Antoch G, Kanja J, Bauer S, Kuehl H, Renzing-Koehler K, Schuette J, et al. Comparison of PET, CT, and dual-modality PET/CT imaging for monitoring of imatinib (STI571) therapy in patients with gastrointestinal stromal tumors. J Nucl Med. 2004;45:357-65.

57. Jager PL, Gietema JA, van der Graaf WT. Imatinib mesylate for the treatment of gastrointestinal stromal tumours: best monitored with FDG PET. Nucl Med Commun. 2004;25:433-8.

58. Stroobants S, Goeminne J, Seegers M, Dimitrijevic S, Dupont P, Nuyts J, et al. 18FDGPositron emission tomography for the early prediction of response in advanced soft tissue sarcoma treated with imatinib mesylate (Glivec). Eur J Cancer. 2003;39:2012-20.

59. Choi H, Charnsangavej C, de Castro Faria S, Tamm EP, Benjamin RS, Johnson MM, et al. CT evaluation of the response of gastrointestinal stromal tumors after imatinib mesylate treatment: a quantitative analysis correlated with FDG PET findings. AJR Am J Roentgenol. 2004:183:1619-28.

60. Choi H, Charnsangavej C, Faria SC, Macapinlac HA, Burgess MA, Patel SR, et al. Correlation of computed tomography and positron emission tomography in patients with metastatic gastrointestinal stromal tumor treated at a single institution with imatinib mesylate: proposal of new computed tomography response criteria. J Clin Oncol. 2007;25:1753-9.

61. Linton KM, Taylor MB, Radford JA. Response evaluation in gastrointestinal stromal tumours treated with imatinib: Misdiagnosis of disease progression on CT due to cystic change in liver metastases. Br J Cancer. 2006;79:e40-4.

62. Desai J, Shankar S, Heinrich MC, Fletcher JA, Fletcher CDM, Tuncali K, et al. Clonal evolution of resistance to imatinib (IM) in patients (pts) with gastrointestinal stromal tumor (GIST): Molecular and radiologic evaluation of new lesions. J Clin Oncol. 2004;22(14S):3010. 
63. Bauer S, Hartmann JT, de Wit M, Lang H, Grabellus F, Antoch G, et al. Resection of residual disease in patients with metastatic gastrointestinal stromal tumors responding to treatment with imatinib. Int J Cancer. 2005;117:316-25.

64. Zalcberg JR, Verweii J, Casali PG, Le Cesne A, Reichardt P, Blay JY, et al. Outcome of patients with advanced gastro-intestinal stromal tumours crossing over to a daily imatinib dose of $800 \mathrm{mg}$ after progression on $400 \mathrm{mg}$. Eur J Cancer. 2005;41:1751-7.
65. Park I, Ryu MH, Sym SJ, Lee SS, Jang G, Kim TW, et al. Dose escalation of imatinib after failure of standard dose in Korean patients with metastatic or unresectable gastrointestinal stromal tumor. Jpn J Clin Oncol. 2009;39:105-10.

66. Demetri GD, van Oosterom AT, Garrett CR, Blackstein ME, Shah MH, Verweij J, et al. Efficacy and safety of sunitinib in patients with advanced gastrointestinal stromal tumour after failure of imatinib: a randomised controlled trial. Lancet. 2006;368:1329-38. 